\title{
Smart Wheel Chair Using Android Based Messenger System
}

\author{
Rahul Das \\ Department of Electronics and Instrumentation Engineering \\ Bengal College of Engineering and Technology, Durgapur, West Bengal, India \\ Amit Agarwal \\ Department of Electronics and Instrumentation Engineering \\ Bengal College of Engineering and Technology, Durgapur, West Bengal, India \\ Amit Kumar Ray \\ Assistant Professor, Department of Electronics and Instrumentation Engineering \\ Bengal College of Engineering and Technology, Durgapur, West Bengal, India
}

\begin{abstract}
Many Wheel-Chair are there in the market some of them are conventional, some are semi- automatic and even some are fully automatic. Day in and day out the development of the wheel chair system are done to provide support to the Differently Abled Persons. Our smart wheel chair system is also a work in this regard to provide help and support to this section of the society. This project aims to develop a Smart Wheel Chair System for the physically handicapped people at an accessible cost. The system is controlled by an Android messenger application and consists of HC-06 Bluetooth module and proximity sensor for the navigational assistance.

Our wheel chair system is cascaded with Bluetooth module to drive the system automatically to the specified position in the horizontal plane. The system is working in accordance with an Android Application. In our work we have used G-chat to connect the Wheel Chair to the Bluetooth module, but in general we can use any Bluetooth messenger application. The system is having two proximity sensors assembled at back and in the front to protect it from any collision. Our wheel chair assembly is having dual password security system in which first password is used to connect the Bluetooth device and the second password is used to get the control over the movement of the wheel chair. Thus making it more secure and advanced.
\end{abstract}

Keywords- Wheel-Chair, Android, Proximity Sensor, Security System, Bluetooth Device.

\section{INTRODUCTION}

Automated wheelchairs that are equipped with sensors and a data processing unit constitute a special class of wheeled mobile robots, termed smart wheelchairs in general literature overviews [1], [2]. Beside general scientific fields of work, such as autonomous navigation approaches, or mapping and self-localization algorithms, the shared spatial reference system between the operator and the smart wheelchair gives rise to certain issues related to user interfaces and shared control problems. For instance, Simpson et al. showed in [3], [4] how to combine discrete driving commands coming from voice control, with navigation assistance provided by reactive navigation approaches. In our work a special type of wheel chair is designed which is equipped with a Bluetooth module which will help to navigate in horizontal plane without any external assistance. The uses the android based messenger application to send the command to drive the Wheel Chair. The Wheel Chair is also equipped with a wireless charging port and proximity sensors to assist it in the automatic navigation. The Fig.1.1. Show the Conventional Wheel Chair present in market, and Fig1.2. Show the Automatic Wheel Chair present in the market.

The project work presented here is structured as follows: - At first we provide a standard description of the Smart wheel chairs present in the market and its working description. Secondly we broadly describe the use of the Bluetooth module in a system to design the Smart Wheel Chair system. At last we provide discussion, analysis and the future prospect of the research in this field. 


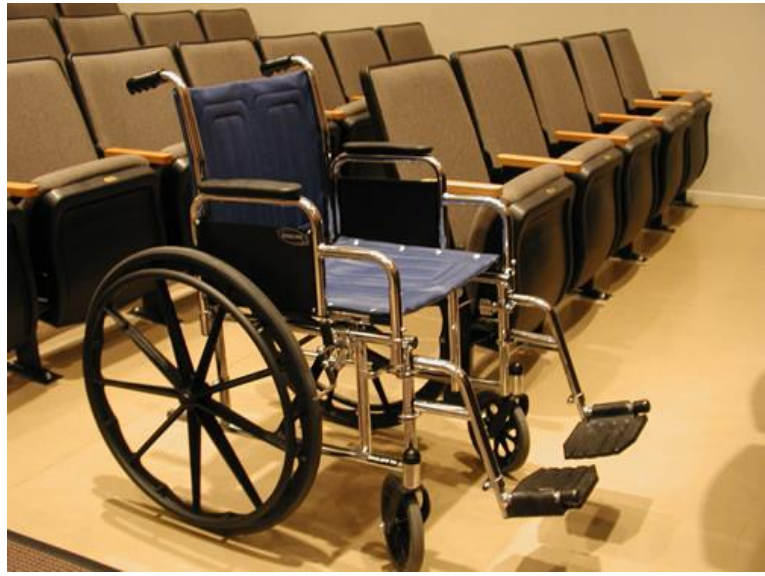

Fig.1.1 Conventional Wheel Chair in the Market

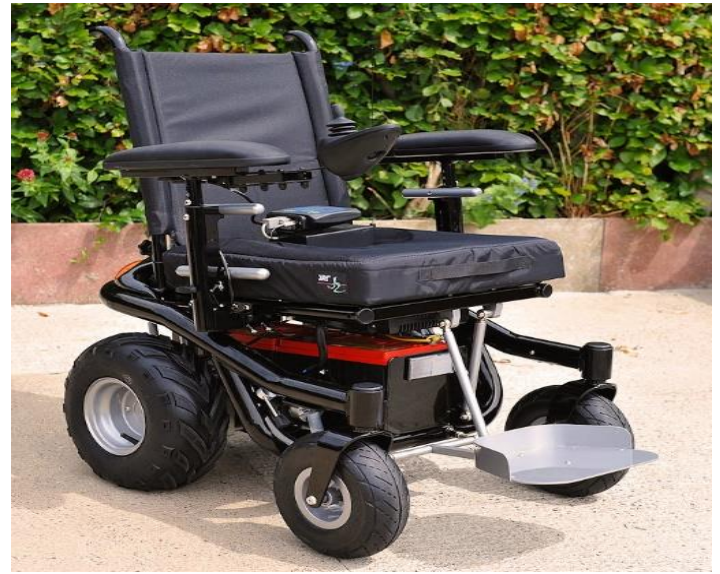

Fig1.2. Fat tyre Joystick Controlled Wheel Chair

\section{BACKGROUND}

\subsection{Smart Wheel Chair System:-}

The paper on smart wheel chair component system by the Simson [5] describe a smart wheel chair typically consisting of either a standard power wheelchair base to which a computer and a collection of sensors have been added or a mobile robot base to which a seat has been attached. Through the year several new assemblies where developed some of them where controlled manually and others where controlled by joystick automatically or semi-automatically. A Canadian company, Applied AI, sells smart wheelchair prototypes for use by researchers, but these devices are not intended for use outside of a research lab. The CALL (Communication Aids for Language and Learning) Centre of the University of Edinburgh, Scotland, has developed a wheelchair with bump sensors and the capability to follow tape tracks on the floor for use within a wheeled-mobility training program. This chair is sold in the United Kingdom (UK), Australia, and the United States by Smile Rehab, Ltd. (Berkshire, UK), as the "Smart Wheelchair.

Many of the smart wheelchairs that have been developed up to date have been integrated with the underlying power wheelchair, requiring significant modifications to function properly. Examples of modifications include adding a object detecting sensors adding a bump sensors etc. Currently, the Smart Wheel Chair Control System, Notre Dame Computer-Controlled Power Wheelchair Navigation System (CPWNS), and the Hephaestus Smart Wheelchair System are the only systems that have been developed as standalone units. The CPWNS are intended to provide the autonomous navigation as they are using the computer vision to detect the special marked place to identify its location as part of an algorithm for accurately reproducing pertained routes. The CPWNS will only work in environments that have been modified to provide navigation cues for the smart wheelchair and will not work in unmodified environments or without pertained routes. The Hephaestus Smart Wheelchair System demonstrated the feasibility of reproducing the desirable obstacle avoidance behaviour developed for the NavChair without requiring modifications to the underlying power. Several of these systems were developed and analysed but their use and implementation in the real market became difficult and costly. 


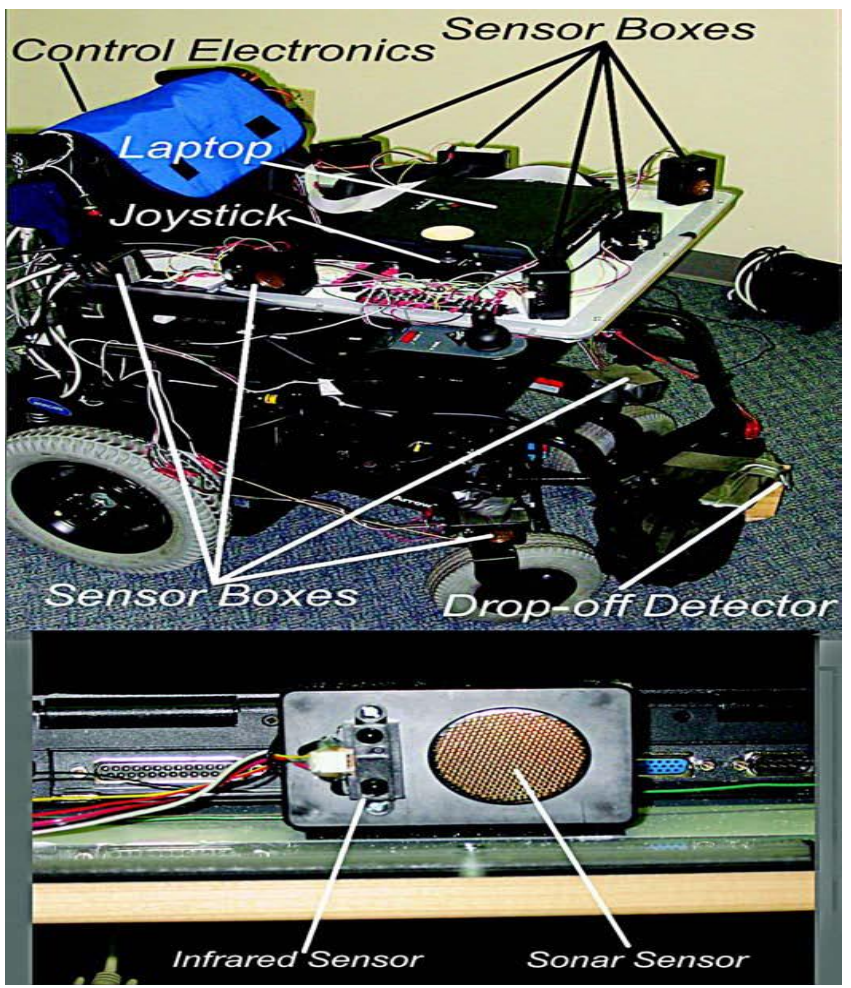

Fig.1.3. Model of Smart Wheel Chair Component System (SWCS)

\subsection{Analysis and Present Day Use}

Census 2001 has revealed that over 21 million people in India as suffering from one or the other kind of disability. This is equivalent to $2.1 \%$ of the population. Among the total disabled in the country, 12.6 million are males and 9.3 million are females. Although the number of disabled is more in rural and urban areas. Such proportion of the disabled by sex in rural and urban areas. Such proportion has been reported between 57-58 percent for males and 42-43 percent females. The disability rate (number of disabled per 100,000 populations) for the country as whole works out to 2130 . This is 2,369 in the case of males and 1,874 in the case of females.

\begin{tabular}{|l|c|c|}
\hline & Population & Percentage \\
\hline Total Population & 1028610208 & $100 \%$ \\
\hline Total disabled Population & 21906769 & $2.1 \%$ \\
\hline
\end{tabular}

Table1. Number of Disabled Population

\begin{tabular}{|l|c|c|}
\hline Type of Disability & Population & Percentage \\
\hline a. In seeing & $10,634,881$ & 1.0 \\
\hline b. In Movement & $6,105,577$ & .6 \\
\hline c. In mental & $2,263,821$ & .2 \\
\hline \multicolumn{2}{|c|}{ Table2. Types of Disability }
\end{tabular}

The above analysis shows the extent of the disability in India. Our Wheel Chair has been customized to provide support to the every section and strata of the society suffering from any physical or mental disability. As the wheelchair can be controlled from any location within the range of the Bluetooth module so it may be used remotely for the paralyzed persons and their motion control.

\subsection{Bluetooth Module}

Bluetooth is a wireless technology standard for exchanging data over short distances (using short-wavelength UHF radio waves in the ISM band from 2.4 to $2.485 \mathrm{GHz}$ ) from fixed and mobile devices, and building personal area networks (PANs). Invented by telecom vendor Ericsson in 1994, it was originally conceived as a wireless alternative to RS-232 data cables. It can connect several devices, overcoming problems of synchronization. 
Bluetooth is managed by the Bluetooth Special Interest Group (SIG), which has more than 25,000 member companies in the areas of telecommunication, computing, networking, and consumer electronics. The IEEE standardized Bluetooth as IEEE 802.15.1, but no longer maintains the standard. The Bluetooth SIG oversees development of the specification, manages the qualification program, and protects the trademarks. A manufacturer must make a device meet Bluetooth SIG standards to market it as a Bluetooth device.

In our experiment we have used the HC-06 module, it is an easy to use Bluetooth SPP (Serial Port Protocol) module, designed for transparent wireless serial connection setup.

Serial port Bluetooth module is fully qualified Bluetooth V2.0+EDR (Enhanced Data Rate) 3Mbps Modulation with complete $2.4 \mathrm{GHz}$ radio transceiver and baseband. It uses CSR Bluecore 04-External single chip Bluetooth system with CMOS technology and with AFH(Adaptive Frequency Hopping Feature). It has the footprint as small as $12.7 \mathrm{mmx} 27 \mathrm{~mm}$. Fig. 1.4 shows the Bluetooth module HC-06.

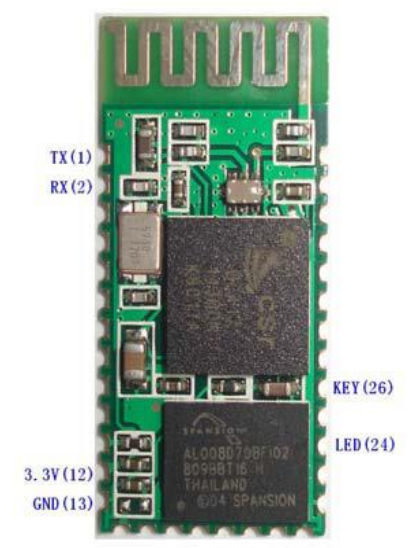

Fig.1.4 Bluetooth module with pin configuration

\section{METHODOLOGY}

\subsection{Circuit Design}

Circuit for this project is designed on the PCB board with Atmel 89C51 microcontroller, the L293M motor driver IC, HC-06 Bluetooth module and some other components as described below in the Table3.

\begin{tabular}{|l|l|l|}
\hline Sl.No & \multicolumn{1}{|c|}{ Components } & \multicolumn{1}{|c|}{ Description } \\
\hline 1. & Resistors & $10 \mathrm{k} \Omega, 1 \mathrm{k} \Omega$ \\
\hline 2. & Capacitor & $0.1 \mu \mathrm{f}, 0.4 \mu \mathrm{f}$ \\
\hline 3. & Voltage regulator & IC 7805 \\
\hline 4. & Oscillator & $12 \mathrm{MHz}$ \\
\hline 5. & Motor Driver IC & L293D (Bidirectional driver) \\
\hline 6. & Microcontroller & ATMEL 89C51(4 KB Flash) \\
\hline 7. & Bluetooth & HC-06 \\
\hline 8. & Power Supply & $12 \mathrm{~V}$ DC Adapter \\
\hline 9. & Software Used & Keil uVision5 \\
\hline 10. & LCD Screen & Blue LCD \\
\hline
\end{tabular}

Table3. Table of Components 
All the components were embedded on the PCB board to make it more cascaded and more sophisticated. Here we are using the $12 \mathrm{~V} \mathrm{DC}$ adapter to drive the system but in the original system we will be using the Wireless Charging assembly to make it more compact and more user friendly.

\subsection{System Design}

The smart wheel chair consist of the HC-06 Bluetooth module, Proximity Sensor for the navigational assistance. The power for the system is drawn from the $12 \mathrm{~V} \mathrm{DC}$ adapter here in the prototype, but in actual model Wireless Charger Will be provided to charge the Wheel Chair without any contact, this will help the differently abled persons to just place the wheel chair above the Charging Assembly and charge the wheel chair batteries. Placement of the different components in the wheel chair casing is shown in the Fig.1.5.

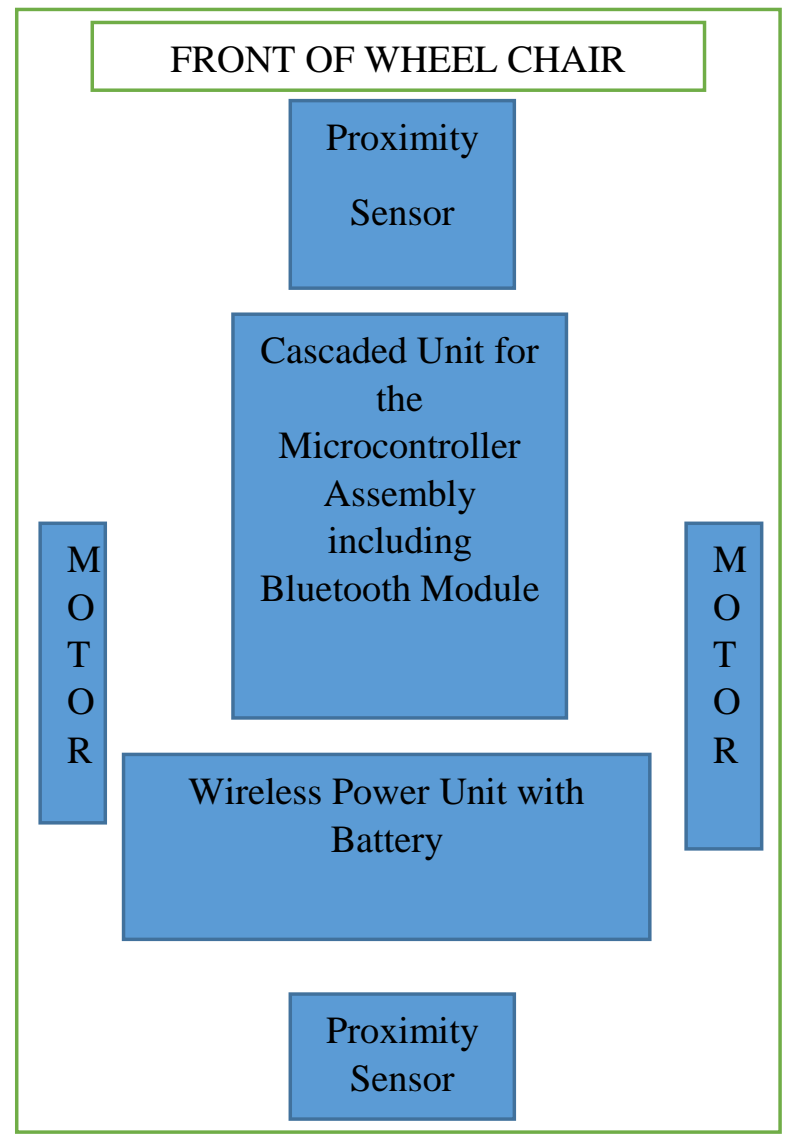

Fig.1.5. System Architecture of the Smart Wheel Chair

\subsection{Transmitting Unit}

The Wheel Chair's microcontroller unit reads the signals from the input device and sends it to the motor driver unit. The motor controller then treats the revised signals as if it has come directly from the input device. The motor connected with motor driver IC-L293D which is subsequently connected with the microcontroller 89C51 (4 KB Flash Programmable and Erasable Read Only Memory and having 128 bytes of RAM). The microcontroller is programmed according to the navigational requirement of the Wheel Chair. The Fig.1.6.provides the transmitting unit of the Wheel Chair Assembly. 


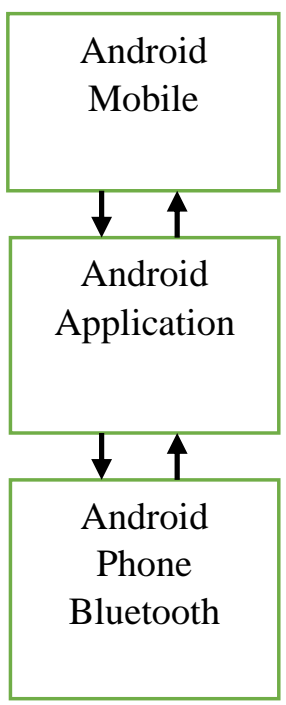

Fig.1.6. Block Diagram of Transmitting Unit of Android Based System

The Android Mobile is used as input. The Application is developed on the Android platform. The Android platform provide a simple messenger type Application which connect with the on board Bluetooth Module (HC06 ) and sends the acknowledge message back to the messenger. When the user sends the message i.e. Forward by typing $(\mathrm{F})$ at that time a string is passed that and then transmitted from the transmission unit to the receiving section through the mobile phone's Bluetooth. At the receiving end the Bluetooth HC-06 accepts the string and sends it to the Microcontroller ATMEL $89 \mathrm{C} 51$ which is a 40 pin programmable interrupt microcontroller. It operates on $5 \mathrm{~V}$ supply and has clock cycle of $12 \mathrm{MHz}$.

\subsection{Receiving Unit}

The microcontroller converts the string into ASCI code and then this code is decoded and according to it the motors are given supply and turned to have linear motion of the wheelchair. Bluetooth module is used for wireless transmission of data, operated on $5 \mathrm{~V}$. Battery of $12 \mathrm{~V}$ is used to drive the wheelchair. Battery is used for the purpose of mobility. DC motors are driven by L293D driver IC, L293D is a dual bridge IC. For forward movement the motors are moved forward and for reverse movement the motors are moved in backward direction. For left movement the left motor is stopped and right motor in forward direction and for right movement the right motor is stopped and left motors are moved in forward direction. Fig. 1.7. shows the block diagram representation of the receiving unit of the Wheel Chair System. 


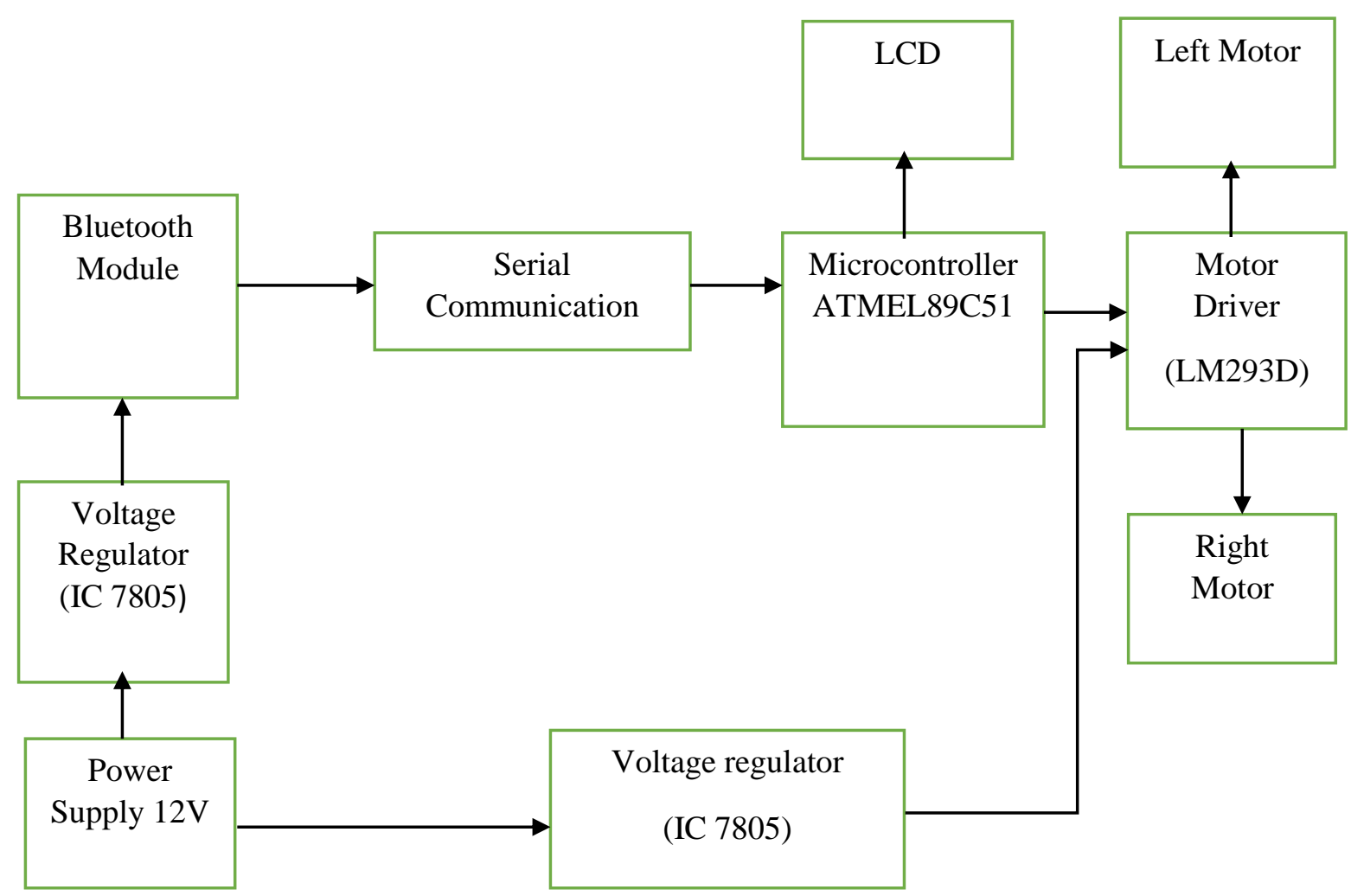

Fig.1.7. Block diagram for Receiver Module in Wheel Chair System

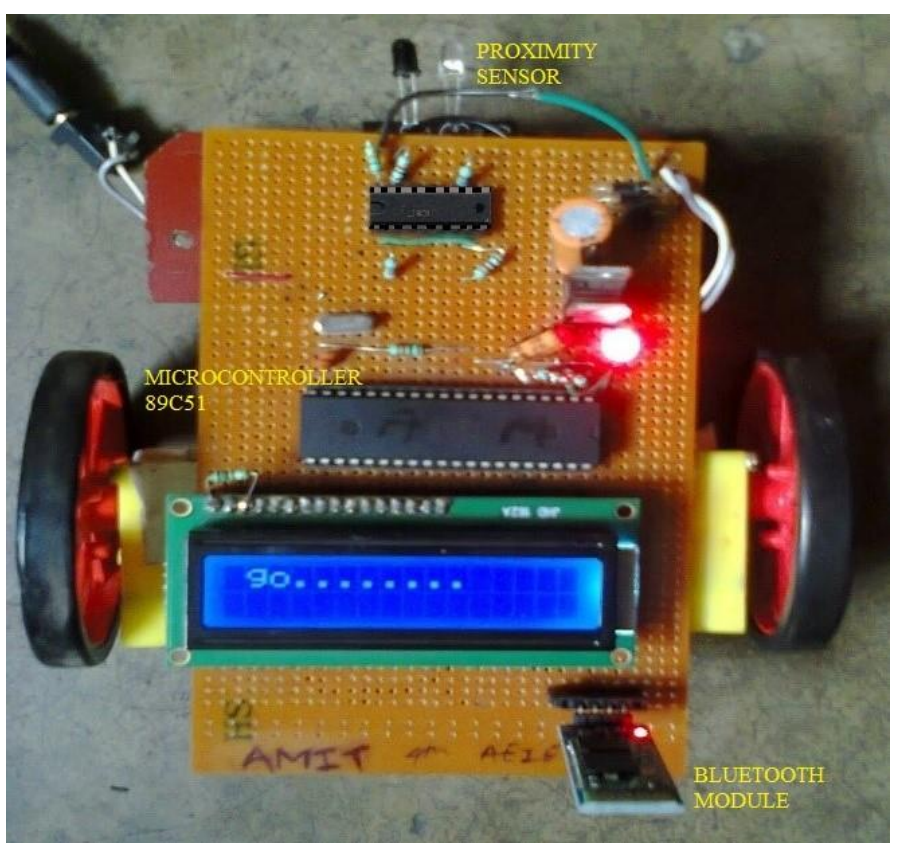

Fig.1.8. Top View of the system Prototype

\subsection{Flow Chart}

The below given flow chart describes the full functioning of our Wheel Chair System. 


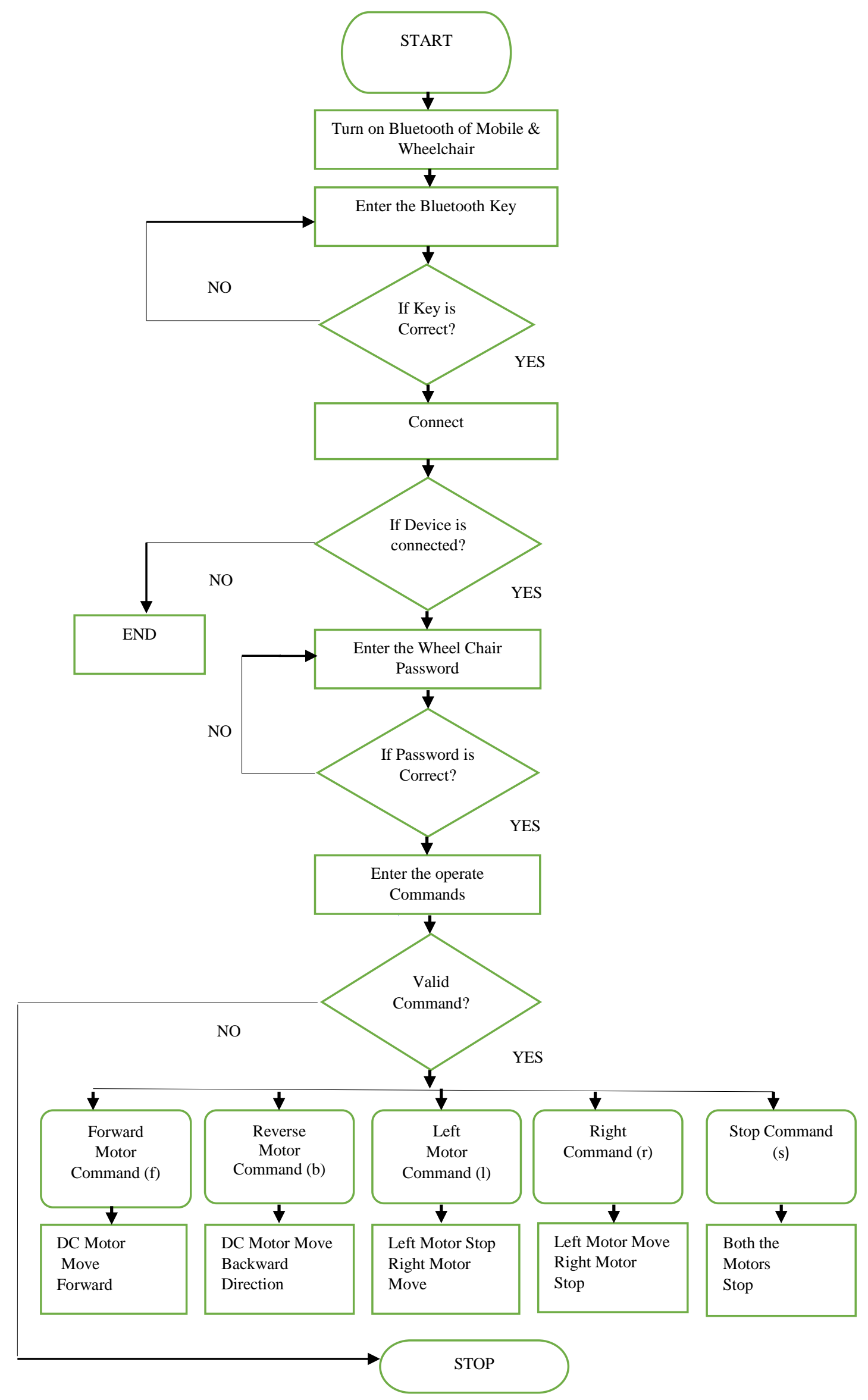


The prototype of the Wheel Chair developed by us follows the above flow chart. Firstly the Bluetooth of the Android based mobile is connected with the Bluetooth of the Wheel Chair System via password, if the first password is correct then, an acknowledge message is received by the Android application. Now the second password is entered to give the commands. After that if we simply type the $\mathrm{f}$ (for the forward) as earlier written in the interfacing Programme then the Wheel Chair will move in forward direction. Similarly when we give the input as b (for Reverse) the Wheel Chair assembly will move in backward direction. Similarly all the other movements are followed as shown in the flowchart.

\subsection{Algorithm}

Switch ON the Bluetooth of both of the device.

If the Key is correct both the device is connected otherwise password has to be entered again.

Enter the Wheel Chair password (second Password) to enter the commands.

If both the device is connected input the Commands to operate the system.

If the requirement is forward then the forward command (f) is entered and all the dc motors are supplied with $12 \mathrm{~V}$ and moved in forward directions for linear movement.

If the requirement is reverse then the reverse command (b) is entered and all the dc motors are supplied with $12 \mathrm{~V}$ and moved in backward directions for linear movement.

If the requirement is to turn left then the left command (l) is entered and thus, left dc motors are stopped and the right dc motors are supplied with $12 \mathrm{~V}$ and the wheelchair moves in left direction.

If the requirement is to turn right then the right command (r) is entered and thus, right dc motors are stopped and the left dc motors are supplied with $12 \mathrm{~V}$ and the wheelchair moves in right direction.

If we have to stop the wheel chair then we must send the Stop(s) message.

\section{DISCUSSION}

The prototype of the Smart Wheel Chair using Android Based Messenger System was designed and tested to check and demonstrate the commercial feasibility. The prototype fulfilled the desired criteria set by us i.e. it can be used with different android applications and even the different android phones were used to conduct the test. This prototype can also be controlled with Laptop PC by using the Bluestacks software and downloading the android application on it and using the same to control the Wheel Chair motion.

The Wheel Chair configuration can also be used with different Wheel Chair configuration in tandem with the Smart system developed by us.

Results from the performance tests demonstrate that the Wheel Chair prototype developed by us can support a wide range of behaviours, including close approach to obstacles without collision. However, the performance test results also demonstrates the different behaviours. Some of the collisions observed during reliability testing could have been avoided if more sensors had been included; however, complete sensor coverage may be impractical in terms of the cost of the final system.

\section{CONCLUSION}

As a conclusion, the objectives of this project have been achieved successfully where in this project the Wheel Chair was automatically controlled by the G-Chat messenger. The development of an android-based wheelchair controller was fully functional base on the objective which are targeted before starting this project. Finally all the combination circuits and the DC motor were embedded to the prototype of wheelchair. This project gives an idea on how to combine all the circuit board, DC motor, and electronics components together in one whole system.

It is obvious that much work need to be done in this field as our work is the first step in providing help and support to the differently abled. The future work will include the GPS module embedded with the Bluetooth module. It will provide specific path tracing facility and a much advanced system to the Wheel Chair system at a very low cost. The Work will also include wireless Charging facility to the wheel chair system, which will be an impetus in the area of automatic navigation in this field. 


\section{REFERENCES}

[1] R. C. Simpson, "Smart wheelchairs: A literature review." Journal of Rehabilitation Research \& Development, no. 4, pp. 423-436.

[2] A. Lankenau and T. Rofer ", "Smart wheelchairs - state of the art in an emerging market," Kunstlic " he Intelligenz. Schwerpunkt Autonome Mobile Systeme, vol. 4, pp. 37-39, 2000.

[3] R. Simpson, S. Levine, D. Bell, L. Jaros, Y. Koren, and J. Borenstein, Lecture Notes in Computer Science: Assistive Technology and Artificial Intelligence. Springer Berling/Heidelberg, ch. NavChair: An assistive

[4] Wheelchair navigation system with automatic adaption, pp. 235-255.

[5] R. Simpson and S. Levine, "Voice control of a powered wheelchair," IEEE Transactions on Neural Systems and Rehabilitation Engineering, vol. 10, no. 2, pp. 122-125, June 2002.

[6] "Smart Wheel Chair Component System" by Richard Simpson, PhD, ATP; Edmund LoPresti, PhD; Steve Hayashi, PhD; Illah Nourbakhsh, PhD; David Miller, PhD

[7] "Voice operated wheelchair" by Jayesh K.Kokate, A.M.Agarkar "international Journal of research in engineering and technology" Volume 3,Issue 2,Feb-2014.

[8] "Controlling an Automatic Wheel Chair via Joystick/Head Joystick supported by smart driving assistance" by Thomas Rofer " and Christian Mandel and Tim Laue.

[9] "Automatic Wheel Chair by Gesture Recognition” by Rakhi A. Kalantri, D.K. Chitre. ISSN: 2249 - 8958, Volume-2, Issue-6, August 2013

[10] "Smart Anroid Wheel Chair” by K. A. A. Kurin, M. H. Mustafa, N. M. Z. Hasim, N. R. M Nuri, A. F. Kadmin, A. Salleha. 